\title{
Le TGV Transmanche : contraintes contextuelles et négociation
}

Pascale Fade

\section{OpenEdition}

\section{Journals}

Édition électronique

URL : http://journals.openedition.org/asp/4315

DOI : 10.4000/asp.4315

ISSN : 2108-6354

\section{Éditeur}

Groupe d'étude et de recherche en anglais de spécialité

\section{Édition imprimée}

Date de publication : 1 octobre 1993

Pagination : 345-354

ISSN : 1246-8185

\section{Référence électronique}

Pascale Fade, «Le TGV Transmanche : contraintes contextuelles et négociation », ASp [En ligne],

2 | 1993, mis en ligne le 05 mars 2014, consulté le 20 avril 2019. URL : http://journals.openedition.org/ asp/4315 ; DOl : 10.4000/asp.4315

Ce document a été généré automatiquement le 20 avril 2019.

Tous droits réservés 


\title{
Le TGV Transmanche : contraintes contextuelles et négociation
}

\author{
Pascale Fade
}

\section{Introduction}

1 En 1994, la mise en service du Tunnel sous la Manche s'accompagnera d'une première ferroviaire, en l'occurrence l'interpénétration de réseaux par la création d'une liaison Paris-Londres-Bruxelles en TGV. Première ferroviaire à différents titres, et en particulier par la présence aux commandes du TGV d'un mécanicien français sur le sol anglais, et vice versa, au lieu de passer la main à la frontière comme c'est habituellement le cas aujourd'hui. Cette nouveauté implique que les mécaniciens des trois pays auront à parler une langue étrangère dans le cadre de leur pratique professionnelle et, en particulier, à bord du TGV pour communiquer avec le personnel au sol. Dans cet exposé, nous analysons les différentes contraintes contextuelles qui ont influé sur la réponse apportée à ce besoin de communication et le rôle de la négociation dans les relations entre tous les partenaires concernés.

\section{Le projet TGV Transmanche}

\subsection{Caractéristiques du projet}

2 Dans notre enseignement aux non-spécialistes, la formation en langue des étudiants est conçue pour répondre à un déficit de compétence par rapport à des objectifs fixés en fonction de besoins à court et moyen terme, besoins évalués en collaboration avec les enseignants de la spécialité. Ceci concourt à déterminer un cadre précis et explicite dans lequel l'enseignant peut mettre en œuvre les pratiques pédagogiques qui lui semblent les plus adaptées pour répondre tant à la spécificité de son public qu'aux objectifs visés. Il doit toutefois composer avec des paramètres tels que le nombre d'heures alloué, les 
effectifs, la plus ou moins grande homogénéité des groupes, paramètres qui peuvent être contraignants.

Dans le cas que nous allons évoquer, la formation des conducteurs de TGV Transmanche, ce cadre précis et explicite n'existait pas. Notre réflexion a été menée dans le cadre d'un projet en cours de réalisation, ce qui signifie que d'une part, les données à intégrer n'étaient pas toutes disponibles dès le départ et, d'autre part, certaines d'entre elles pouvaient subir des modifications ou se transformer au fur et à mesure de l'avancement du projet. Enfin, aucune expérience similaire dans le monde ferroviaire ne pouvait servir de modèle ou de référence. Notre travail a donc consisté, entre autres, à définir le cadre le plus précis et le plus explicite possible, c'est-à-dire la nature et le degré des compétences à acquérir, puis de déterminer la réponse-formation adéquate.

\subsection{Problématique}

4 La demande initiale formulée par la SNCF auprès de CITE Langues ${ }^{1}$ ne se limitait pas à une demande de formation. Elle peut être résumée de la manière suivante : dans le cadre de la mise en service des lignes de TGV Transmanche, les conducteurs auront à parler anglais en particulier au cours du trajet avec les personnels au sol britanniques, et ce par liaison radio, mais aussi une fois arrivés à Londres (en gare de Waterloo et durant leur période de repos). Il convient donc de leur proposer une formation adaptée tant aux contextes d'utilisation qu'aux exigences de sécurité (étant entendu qu'une formation lourde visant le « bilinguisme » n'est pas envisageable).

5 La formulation de la demande suggérait il est vrai une formation mais, avant de pouvoir l'envisager, il était pour le moins nécessaire d'obtenir de plus amples précisions quant au contexte ferroviaire, aux enjeux, aux situations d'utilisation de la langue et à la nature même des communications envisagées.

6 Pour faire court, disons que, schématiquement, deux réponses ont été apportées :

- une formation qui répond aux besoins de communication recensés dans les situations «en gare de Waterloo » et « en période de repos », après analyse des besoins et élaboration d'une ingénierie de formation;

- la création d'un système de communication orale formalisée pour la communication radio à bord du TGV.

7 Nous développerons plus particulièrement les principales contraintes contextuelles qui ont pesé sur la deuxième réponse. Ces contraintes sont les plus significatives du projet et englobent en grande partie celles relatives à la première.

\section{Contraintes contextuelles}

Il est nécessaire tout d'abord de repréciser en quelques mots le contexte qui a conduit à la création d'une communication orale formalisée. À bord du TGV, lors du déroulement «normal » du trajet, aucune communication orale n'est nécessaire entre le conducteur et le personnel au sol. Ce n'est que dans des situations qui perturbent le bon déroulement ou 
la sécurité du trajet que le conducteur et le personnel au sol entrent en contact radio pour définir les comportements à avoir et/ou les mesures à prendre.

Les facteurs les plus exigeants qui ont influé sur la réflexion et conduit à l'élaboration d'une communication orale formalisée sont les suivants :

- le recours à la communication orale sera rare ;

- la communication sera toujours liée à un enjeu absolu, la sécurité ;

- la communication s'établit entre deux interlocuteurs dont l'un parle une langue étrangère (le conducteur) et l'autre sa langue maternelle (le personnel au sol).

Dans notre réflexion initiale menée en collaboration avec les linguistes britanniques, il est intéressant de noter que nous avons rapidement partagé le même point de vue, et qu'un accord sur le concept même de communication orale formalisée a été atteint sans difficulté majeure. C'est dans l'application des principes linguistiques au contexte ferroviaire que nous avons rencontré l'essentiel des difficultés : dans l'élaboration des dialogues formalisés qui constituent cette communication orale formalisée, certaines données ferroviaires, inhérentes au projet ou spécifiques à la SNCF, se sont révélées difficiles à intégrer. Si les partenaires ferroviaires ont été les premiers concernés par les problèmes soulevés, ces problèmes ont rejailli, par ricochet, sur notre travail.

\subsection{Contraintes liées au projet}

11 Le projet de liaison Paris-Londres-Bruxelles via le tunnel sous la Manche rassemble plusieurs partenaires qui, certes, partagent le même objectif mais ne partagent pas les mêmes conceptions ferroviaires, ni la même langue, ni la même culture. Ces différences ont inévitablement un impact sur la collaboration entre la SNCF pour la France, EPS (filiale de BR) pour la Grande-Bretagne, la SNCB pour la Belgique et Eurotunnel pour le tronçon sous la Manche.

12 L'objectif commun est qu'un mécanicien, quelle que soit sa nationalité, conduise un TGV sur la totalité du parcours, quel que soit le réseau. Cet objectif impose, dans une première phase, de bien connaître le réseau des autres partenaires afin, dans une deuxième phase, de parvenir à une harmonisation. Celle-ci impliquant des ajustements et des modifications du système ferroviaire propre à chaque pays. Ces deux étapes, la connaissance des réseaux et l'harmonisation, vont se réaliser par le biais de la négociation, au sens linguistique du terme : la négociation réunit ici des interlocuteurs qui partagent le même objectif de communication et qui sont disposés à instaurer une coopération pour parvenir à un accord au plan des significations (compréhension des intentions de communication et des informations). Par conséquent, ils vont favoriser la création des conditions requises pour que l'objectif soit atteint. Entre autres, ils vont mettre en œuvre des stratégies destinées à faciliter la compréhension des informations transmises; stratégies qui contribuent à l'établissement d'un contexte de référence commun, c'est-à-dire partagé par tous les partenaires.

Dans le cas particulier, le contexte de référence commun correspond au descriptif de l'ensemble des réseaux. Si la volonté d'établir ce contexte de référence commun est manifeste, les différences ferroviaires, culturelles et linguistiques mentionnées précédemment seront toutefois autant de handicaps à surmonter pour y parvenir.

14 Dans le domaine ferroviaire, la connaissance du réseau étranger pour les partenaires français et anglais a nécessité la traduction de la «bible du mécanicien » (Rule Book en 
anglais, PGM - Prescriptions Générales concernant le Mécanicien - en français). Lors de cette traduction, ainsi que dans les nombreuses réunions de travail rassemblant les spécialistes des trois pays, la compréhension des informations a été considérablement gênée par le manque de référents d'un système à l'autre, que ce soit dans le domaine de la signalisation, des instructions de sécurité... Il n'est en effet pas évident de faire comprendre une réalité qui n'existe pas pour l'autre et, pour cela, de trouver les mots justes, les explications adéquates ou les illustrations pertinentes. Et inversement, il n'est pas évident, dans les mêmes conditions, de comprendre le discours de l'autre. En d'autres termes, chaque spécialiste a dû intégrer à son champ de référence des notions nouvelles ou des concepts différents des siens, pour qu'ils parviennent tous à un contexte commun, précis et explicite.

Dans le domaine culturel ou de l'organisation interne de l'entreprise, les différences sont nombreuses - que ce soit dans la méthodologie d'approche, le rythme d'avancement, la perception du projet - autant de différences qui influent sur le déroulement des réunions de travail. Par exemple, une conception différente des postes de travail et de la hiérarchie ne favorise pas la rencontre d'homologues à proprement parler, et ceci peut nuire à la transmission des informations et éventuellement à leur qualité. Un responsable " sécurité » en France n'a pas nécessairement son équivalent en Grande-Bretagne, et l'inverse peut être vrai pour un poste britannique précis. Une demande d'informations peut alors nécessiter l'intervention de plusieurs personnes partageant le domaine de compétences d'un demandeur unique.

Enfin, les différences linguistiques vont engendrer des difficultés qui se superposent aux précédentes. La présence d'interprètes lors des réunions de travail va naturellement apporter une aide non négligeable. Mais dans cette spécialité, comme dans toute spécialité, seul l'initié détient véritablement la connaissance et, en particulier, le sens des mots : le risque d'une simplification ou d'une approximation reste bien présent. De plus, le «jargon» ferroviaire est omniprésent dans chaque langue. Pour le profane, un " tournesol », par exemple, n'évoque pas systématiquement un répétiteur de signal, et les abréviations PN, PK ou autres ne sont pas immédiatement «transparentes »... Les pièges linguistiques sont nombreux et ne favorisent ni l'échange ni la compréhension des informations.

\section{Autres exemples :}

- la traduction littérale d'une expression peut être source d'erreur: "wrong direction» en anglais correspond à « contre-voie » en français et non pas à « fausse direction », cependant " fausse direction » existe bien mais a une autre signification.

- L'utilisation de termes voisins dans les deux langues peut être trompeuse, car ils ne recouvrent pas le même concept ou la même réalité : «caténaire » en français est un terme générique qui correspond à l'ensemble du dispositif alors que «catenary» fait uniquement référence à l'élément porteur.

18 Si la négociation, malgré les difficultés évoquées, est efficace pour parvenir à un contexte de référence commun, l'harmonisation est elle plus délicate à construire. La raison essentielle est que, dans cette phase, l'enjeu présent a des conséquences non négligeables : des modifications et des remises en cause de procédures résulteront des choix effectués. Chacun va donc défendre ses options, tenter d'imposer ses vues, essayer de convaincre l'autre de la meilleure adéquation de son système et aussi fixer les limites qu'il ne peut (ou ne veut) franchir. En d'autres termes, c'est une négociation, au sens stratégique ou politique cette fois, qui vient se greffer à la première. Vu l'importance de 
l'enjeu, cette négociation sera longue et va globalement freiner l'avancement du projet. Elle va également mettre à jour des différences passées inaperçues initialement qui vont relancer le débat et d'autant plus ralentir la progression.

\subsection{Contraintes liées à la SNCF}

En tant que consultants de la SNCF, nos interlocuteurs privilégiés sont bien évidemment les responsables ferroviaires français. Ce sont eux qui ont défini le cahier des charges à respecter dans notre réponse linguistique et aussi imposé des contraintes, inhérentes à l'entreprise, sur son élaboration.

20 Pour nous, il était indispensable d'approfondir nos connaissances ferroviaires et d'obtenir des informations techniques, et seuls les spécialistes pouvaient nous renseigner. Toutefois, il était nécessaire de bien cibler nos demandes pour qu'elles soient satisfaites, le spécialiste n'étant pas toujours à même d'évaluer la nature des informations à apporter pour une bonne compréhension de l'autre. En outre, inconsciemment, il peut ne pas être favorable à une intrusion dans son domaine et il peut souhaiter se limiter à la transmission d'informations qu'il juge nécessaires et suffisantes. L'obtention des informations n'a donc été que progressive, relancée par nous au fur et à mesure des besoins ressentis. Mais elle a aussi été liée, comme nous l'avons vu précédemment, à l'évolution de la connaissance des réseaux étrangers de la part de nos interlocuteurs et globalement à l'avancement du projet.

21 L'intervention de plusieurs services de la SNCF, le service "technique», le service "sécurité " et le service "formation", tous partie prenante dans le projet, a mis en évidence des approches différentes. Les champs de responsabilités n'étant pas les mêmes d'un service à l'autre, chacun a exprimé, fort logiquement, un point de vue tenant compte essentiellement de sa réalité. Par exemple, le service «formation » a rapidement adhéré au concept de la communication orale formalisée qui, par ses règles, apportait une harmonisation dans la formulation des procédures de sécurité et par ailleurs facilitait leur apprentissage. Le service "sécurité » quant à lui était soucieux de préserver les procédures en vigueur sur le réseau français. Il n'a adhéré aux modifications proposées qu'au terme d'une négociation sur les points de divergence, dans la mesure où ces modifications représentaient un compromis compatible avec les procédures existantes.

Enfin, l'organisation de l'entreprise, sa politique interne et en particulier sa gestion des ressources humaines, ont défini les exigences du cahier des charges initial.

Le premier paramètre a été le facteur temps : la hiérarchie dans les priorités de formation plaçait naturellement la formation technique ferroviaire au premier rang et la durée de la formation linguistique devait donc s'inscrire dans le temps-formation laissé disponible. Les personnels assurant dans le même temps un service partiel, il s'agissait de concilier les différents impératifs dans le délai fixé initialement par la date de mise en service du TGV.

24 Deuxième paramètre, les caractéristiques individuelles des personnes à former: les conducteurs sélectionnés au sein de l'entreprise présentaient des profils variés, et leur potentiel d'apprentissage ainsi que leur motivation étaient loin d'être homogènes. Leur formation initiale correspondant à CAP, Bac ou "formation interne", les acquis linguistiques antérieurs étaient donc très divers. Précisons au passage que le critère « langue » n'a pas été un critère discriminatoire dans la sélection des conducteurs. Par 
ailleurs, il convenait d'éviter que les compétences requises en langue ne constituent un facteur de «stress » psychologique.

Dernier paramètre, le caractère reconductible du plan de formation. La formation des conducteurs de TGV devait se réaliser en plusieurs vagues annuelles. Le plan de formation conçu pour les premiers conducteurs se devait donc d'être reconductible pour les suivants, tant sur le plan de la durée et du contenu de la formation que des moyens financiers. En ce sens le budget initial engageait donc l'avenir.

\section{Contraintes et libertés du linguiste}

Dans la solution retenue, la communication orale formalisée entre les conducteurs de TGV et les personnels au sol, la collaboration entre CITE Langues et les linguistes britanniques était une condition impérative pour parvenir à un système identique des deux côtés de la Manche. Elle a rapidement abouti à un accord sur les principes linguistiques à appliquer dans ce système.

Toutefois, dans la phase plus autonome de réflexion où chacun travaillait sur sa langue, la qualité de la collaboration est devenue fortement dépendante des relations entre la compagnie ferroviaire et son partenaire linguistique. En effet, chaque équipe était tributaire des réactions de son partenaire ferroviaire vis-à-vis du système proposé, de sa compréhension et de son adhésion plus ou moins rapide. De plus, un système commun étant obligatoire, les compagnies devaient décider ensemble de son acceptation, le plus convaincu ayant à charge de convaincre l'autre. Une fois le système retenu, l'implication du partenaire ferroviaire était toutefois essentielle et un engagement inégal de part et d'autre a eu pour conséquence un rythme de progression, côté linguistes, très différent. La collaboration dans ces conditions, est devenue moins étroite, plus irrégulière.

Tout en respectant le cahier des charges initial et les contraintes contextuelles, c'est dans ses propositions de réponse que le linguiste, en l'occurrence le consultant-linguiste, va retrouver sa liberté. En effet la réflexion s'inscrit dans son champ de compétence et il va fixer, en fonction de critères relatifs à son domaine, les règles à appliquer. Cet espace de liberté correspond à la mise en œuvre de son « savoir » et savoir-faire et c'est d'ailleurs la raison pour laquelle il a été consulté. On lui reconnaît une autorité linguistique qu'il va exercer pour élaborer sa réponse.

Cependant, le rôle de consultant externe ne nous confère pas un droit de décision ou la possibilité d'imposer nos choix, du simple fait de notre compétence reconnue. Notre rôle, si nous sommes convaincus de la justesse de notre réflexion, va donc consister à démontrer le caractère pertinent de notre réponse en appliquant une stratégie appropriée. Toutefois, certaines propositions peuvent rencontrer une résistance ou aller à l'encontre de situations bien établies, et faire l'objet d'une remise en cause. Il s'agit alors de bien comprendre les arguments avancés pour rejeter telle ou telle proposition, mais aussi de présenter de façon explicite les implications de certains changements. C'est alors par étapes successives qu'une négociation va progresser. Tout d'abord, chacun va réintroduire dans sa réflexion les remarques retenues comme pertinentes et étudier les répercussions sur son domaine. Ensuite, il s'agit d'exposer, le cas échéant, les incompatibilités mises à jour, la perte de cohérence qui résulterait des éventuelles modifications ou les changements possibles. C'est après un certain nombre d'allers et retours que se fait le choix final et, selon les cas, le «linguistique » l'emporte sur le 
«ferroviaire » ou inversement, après prise en considération de tous les effets secondaires induits. Notre rôle est d'argumenter en faveur des solutions qui nous paraissent les plus favorables à la qualité globale.

\section{Conclusion}

Outre les facteurs et paramètres qui ont orienté le choix d'un système de communication orale formalisée entre conducteurs de TGV et personnels au sol, nous venons de rapporter les principales contraintes contextuelles qui ont pesé sur son élaboration. La définition de contrainte dans le dictionnaire Le Robert indique "entrave à la liberté d'action ». Dans notre expérience, les contraintes n'ont pas été obligatoirement ressenties comme négatives ou frustrantes. En effet, bon nombre d'entre elles ont contribué à délimiter le cadre précis et explicite d'intervention auquel nous faisions référence au début de notre exposé. La problématique initiale devait s'affiner, et une réponse adaptée et pertinente ne pouvait être apportée que par la prise en compte des exigences contextuelles, aussi contraignantes fussent-elles.

\section{ANNEXES}

\section{Annexe : Compte-rendu des discussions}

Plusieurs questions concernent la formation des mécaniciens

La formation est assurée par les Centres de Langues des GRETA les plus proches des lieux de recrutement et de formation ferroviaire, en l'occurrence Lille et Paris-Nord. CITE langues est concepteur de la formation et il s'agit donc d'un transfert entre CITE langues et les formateurs des GRETA. Des rencontres préalables à la formation ont permis d'expliquer les objectifs et les choix qui ont conduit à l'élaboration du référentiel de formation, les formateurs étant chargés de sa mise en œuvre pédagogique. La première phase de formation est en cours et concerne 4 « vagues » de mécaniciens. Elle répond aux besoins identifiés en période de repos et en gare de Waterloo. Elle inclut des séjours linguistiques axés sur les besoins professionnels (visite de la gare de Waterloo, rencontre des homologues britanniques...). La deuxième phase, qui concerne la communication orale à bord du TGV, n'est pas encore entamée. La pratique sur le simulateur de conduite sera un élément clé de cette formation. Il est prévu d'enregistrer les dialogues avec une variété d'accents afin « d'entraîner l'oreille » des mécaniciens à des accents différents.

Précisions sur l'élaboration des dialogues qui constituent la communication orale formalisée

Chaque pays élabore, dans sa langue, l'ensemble des dialogues formalisés et un transfert s'effectuera ensuite pour permettre la formation des personnels concernés. CITE langues élabore donc les dialogues en français et les linguistes du Consortium en anglais. Dans l'utilisation de la compréhension orale formalisée, il est important de considérer que l'un des interlocuteurs (le mécanicien) utilise une langue étrangère alors que l'autre (le 
personnel au sol) utilise sa langue maternelle. Le personnel au sol, s'il a l'avantage de s'exprimer dans sa langue, doit toutefois respecter strictement le déroulement des dialogues afin de ne pas perturber la communication. Il devra s'assurer aussi que son débit de parole est mesuré et son élocution claire.

\section{Extension du problème à la Belgique et au futur réseau européen}

La liaison Bruxelles-Londres via le Tunnel implique les partenaires belges au même titre que les Français et les Anglais. Le futur réseau européen devrait bénéficier de l'expérience capitalisée.

\section{NOTES}

1. CITE Langues (Conseil Ingénierie Technologie Étude en Langues) 28, rue de Saurupt, 54000 Nancy.

\section{RÉSUMÉS}

En 1994, des mécaniciens français conduiront le TGV sur le sol britannique, et vice versa, première ferroviaire due au TGV Transmanche. Les contraintes contextuelles (interpénétration de réseaux, partenaires nombreux, sécurité, données techniques partiellement non maîtrisées...) influent fortement sur l'élaboration d'un système de communication orale entre mécaniciens et personnels au sol. La définition linguistique de ce système passe par des négociations constantes entre les différents partenaires. La liberté du linguiste, dans cet environnement de contraintes, existe-t-elle?

In 1994, high-speed trains will run from Paris to London, and vice versa, via the Channel Tunnel with no change of driver. The interpenetration of railway networks, the number of parties involved, safety requirements... all have a restricting effect on the development of a verbal communication system to be used between drivers and signalmen. The linguistic definition of this system is subjected to constant negotiation between the parties concerned. In such a context, to what extent is the linguist affected in his work?

\section{INDEX}

Mots-clés : communication orale, négociation, TGV Transmanche

Keywords : oral communication, negotiation, Paris-London high-speed train

\section{AUTEUR \\ PASCALE FADE}

Université Nancy 2, consultante pour CITE Langues. pascale.fade@univ-lorraine.fr 